\title{
On the temperature dependence of the nonexponentiality in glass-forming liquids
}

\author{
Daniele Cangialosi, ${ }^{1, a)}$ Angel Alegría, ${ }^{1,2}$ and Juan Colmenero ${ }^{1,2,3}$ \\ ${ }^{1}$ Facultad de Química, Centro de Física de Materiales Centro Mixto (CSIC-UPV/EHU), \\ Apartado 1072, 20080 San Sebastián, Spain \\ ${ }^{2}$ Departamento de Física de Materiales, Universidad del País Vasco (UPV/EHU), \\ Apartado 1072, 20080 San Sebastián, Spain \\ ${ }^{3}$ Donostia International Physics Center, Paseo Manuel de Lardizabal 4, 20018 San Sebastián, Spain
}

(Received 16 January 2009; accepted 22 February 2009; published online 23 March 2009)

\begin{abstract}
Using a simple mathematical formalism, we show that temperature dependent nonexponential relaxation found in glass-forming liquids and amorphous polymers, often resulting in a decrease in the stretching exponent when decreasing temperature, can be suitably described assuming the combination of an intrinsic stretched response and the existence of temperature independent heterogeneities. The effect of the latter is incorporated by assuming a Gaussian distribution of Vogel temperatures. Comparison with experimental data of a large number of glass formers showed that this approach is able to quasiquantitatively describes the temperature dependence of the stretching exponent using the width of the distribution as the single fitting parameter. According to this approach, the rapidity of the decrease in the stretching exponent with decreasing temperature depends not only on the magnitude of the standard deviation of Vogel temperatures but also on the value of the intrinsic stretching exponent and on the fragility of the glass former. The latter result is able to rationalize, at least partially, the empirical correlation between the fragility and the stretching exponent at $T_{g}$. (C) 2009 American Institute of Physics. [DOI: 10.1063/1.3098904]
\end{abstract}

\section{INTRODUCTION}

The dynamics of glass-forming liquids and amorphous polymers generally display nonexponential behavior. This reflects on the decay of the relaxation function associated to the glass transition. This behavior is conveniently expressed by the well-known Kohlrausch-Williams-Watts (KWW) equation: ${ }^{1,2}$

$$
\Phi(t)=\exp \left[-\left(t / \tau_{\mathrm{KWW}}\right)^{\beta_{\mathrm{KWW}}}\right],
$$

where $\Phi(t)$ is the relaxation function, $t$ is the time, $\tau_{\mathrm{KWW}}$ is the KWW relaxation time, and $\beta_{\mathrm{KWW}}<1$ is the stretching exponent.

The nonexponential relaxation of glass-forming liquids and amorphous polymers has attracted the attention of a large number of scientists in the past years (see, e.g., Ref. 3, and references therein), also in view of its empirical relation with another outstanding signature of the glass transition, ${ }^{4}$ namely, the non-Arrhenius slowing down of the dynamics associated to the structural relaxation. ${ }^{5}$ Among the features concerning $\beta_{\mathrm{KWW}}$, the experimental evidence that this parameter decreases when approaching the glass transition temperature $\left(T_{g}\right.$, generally defined as the temperature where the relaxation time is about $100 \mathrm{~s}$ ) from above is one of the most notable. Although such a decrease is in some cases not observed for some glass-forming liquids verifying the timetemperature superposition, ${ }^{6-9}$ the increasing nonexponential behavior when approaching the $T_{g}$ from above has been often attributed to the evolution of dynamical heterogeneities

${ }^{a)}$ Electronic mail: swxcacad@sw.ehu.es. occurring below about $1.2 T_{g} .{ }^{10-14}$ According to this interpretation the stretching of the relaxation function associated to the glass transition would be mainly related to the heterogeneous nature of glass-forming liquids and amorphous polymers, ${ }^{15}$ rather than being an intrinsic peculiarity of each relaxing unit in the glass former. Nonetheless, the connection between heterogeneous dynamics and nonexponential relaxation, and their temperature evolution, in glass-forming liquids is still far from being clarified. ${ }^{16}$

In this work, we develop a simple mathematical formalism that shows that the temperature dependence of the stretching exponent $\beta_{\mathrm{KWw}}$ of a large number of glass formers, both polymeric and nonpolymeric, can be conveniently reproduced assuming an intrinsic nonexponential relaxation, which would be reflected in the high temperature behavior, and temperature independent dynamical heterogeneities. The latter can be suitably described by a distribution of Vogel temperatures [and consequently of $T_{g}(s)$ ], i.e., the temperature where the structural relaxation time is predicted to diverge according to the Vogel-Fulcher-Tamann (VFT) phenomenological equation. ${ }^{17}$ Apart from discussing the real connection between the temperature evolution of heterogeneities and the degree of stretching, within our approach we also provide a link between the fragility of the glass former and its stretching exponent at $T_{g}$, thus rationalizing, at least partly, the empirical finding of Böhmer et al. ${ }^{4}$ Despite the massive number of glass formers included in their analysis including materials possessing a wide variety of chemical structures-leading to a large scattering in their analysisthey found a tendency to an approximate inverse proportionality between the fragility and the degree of stretching. 


\section{MATHEMATICAL FORMALISM}

In this section of this paper, we present the mathematical formalism of our approach to the description of the temperature dependent nonexponentiality. We employ the VFT equation to describe the temperature dependence of the characteristic relaxation time $(\tau)$ of the $\alpha$ process: ${ }^{17}$

$$
\tau=\tau_{0} \exp \left(\frac{D T_{0 \text { (av) }}}{T-T_{0 \text { (av) }}}\right),
$$

where $\tau_{0}, D$, and $T_{0 \text { (av) }}$ are, respectively, the pre-exponential factor, a glass-former specific parameter, and the average Vogel temperature.

We assume that the structural relaxation is nonexponential in nature-as suggested by experiments ${ }^{16}$ and predicted by notable theories of the glass transition ${ }^{18,19}$ - and that, due to some dynamical heterogeneities, which might have spatial counterparts (e.g., density spatial fluctuations) ${ }^{15}$ a distribution of Vogel temperatures $g\left(T_{0}\right)$ exists. We also assume for simplicity that such a distribution is Gaussian:

$$
g\left(T_{0}\right)=\frac{1}{\delta T_{0} \sqrt{2 \pi}} \exp \left[-\frac{\left(T_{0}-T_{0(\mathrm{av})}\right)^{2}}{2 \delta T_{0}^{2}}\right],
$$

where $\delta T_{0}$ is the standard deviation of the distribution of Vogel temperatures.

Assuming a distribution of Vogel temperatures implies that the lifetime of heterogeneities is larger than that of the structural relaxation time. However, this assumption still holds even if the lifetime of such heterogeneities is of the order of the relaxation time of the structural relaxation, as indicated by several recent experiments. ${ }^{10,11,13,15,20}$ These are based on multidimensional NMR and are able to provide the typical time scale of heterogeneities, which is actually found to be of the order or larger than the structural relaxation time. Our approach can still be safely employed even if exchange between relatively fast and slow regions occurs in the time scale of the structural relaxation, since in this case the ensemble probed experimentally generally maintains the average distribution of environments. In other words, the exchange between environments in the time scale of heterogeneities can be considered as a dynamic equilibrium.

The soundness of the assumption of a distribution of Vogel temperatures will be discussed when comparing our approach with available experimental stretching exponents. Due to the distribution of Vogel temperatures, there would also be a temperature dependent distribution of relaxation times $G(\ln \tau)$ given by

$$
G(\ln \tau)=\frac{g\left(T_{0}\right)}{\frac{d \ln \tau}{d T_{0}}}=g\left(T_{0}\right) \frac{\left(T-T_{0}\right)^{2}}{D T} .
$$

Thus, the relaxation function of the system at a given temperature-obtained from different experiments providing the correlation function for molecular dynamics - can be obtained integrating the following expression:

$$
\Phi(t, T)=\int_{-\infty}^{+\infty} G(\ln \tau(T)) \exp \left[-\left(\frac{t}{\tau(T)}\right)^{\beta_{0}}\right] d \ln \tau,
$$

where $\beta_{0}$ is the intrinsic exponent (unrelated to the presence of heterogeneities), which can be experimentally determined in the high temperature region, ${ }^{21}$ where the heterogeneities are not expected to play any major role. It has to be remarked that such a high temperature limit, apart from depending on the specific glass former, can also depend on the technique employed, i.e., on the relaxation function measured. The observed macroscopic relaxation function is, in fact, related to a specific molecular time correlation function ${ }^{22}$ that, in the case of dielectric relaxation spectroscopy widely employed in this work, is the dipole orientational correlation function. ${ }^{23}$ As notable examples of experimental techniques providing different degree of stretching in the same glass former, we mention the case of $o$-terphenyl ${ }^{24,25}$ (OTP) and that of poly(vinyl acetate). ${ }^{26}$ In the former case a marked difference between the stretching exponent measured by Fabry-Pérot interferometry ${ }^{24}$ - a technique based on light scattering — and that obtained by neutron scattering ${ }^{25}$ can be observed. Both techniques provide information on density decorrelation although on different spatial lengths. A similar mismatch is observed when comparing dielectric and neutron scattering experiments in poly(vinyl acetate) performed in the same temperature range, namely, where the same relaxation time is detected. ${ }^{26}$ In this case, such a mismatch would arise from the different correlation functions accessed by the two techniques, namely, the decorrelation of dipoles for dielectric spectroscopy and that of density for neutron scattering. However, despite these differences, our approach can still be formally employed for each relaxation function.

Within our approach, from the knowledge of the VFT parameters-allowing the description of the temperature dependence of the structural relaxation time and that of the high temperature limit of the stretching exponent $\beta_{0}$-the relaxation function can be calculated at any temperature, having $\delta T_{0}$ as the only adjustable parameter. The resulting function can be subsequently fitted through the stretched exponential [Eq. (1)] and the $\beta_{\mathrm{KWW}}(T)$ parameter extracted. This can be compared to stretching exponent determined experimentally. The breadth of the Gaussian distribution of Vogel temperatures $\delta T_{0}$ is that generating a stretching exponent that best reproduces the experimental one.

In Fig. 1(a), typical relaxation functions at different temperatures generated through Eq. (5) are presented (points) together with the fitting through the KWW function (continuous lines). Log-time derivatives of the relaxation functions, promptly comparable to dielectric loss data once Fourier transformed to the frequency domain, ${ }^{27}$ are shown in Fig. 1(b). As an example the VFT parameters of poly(vinyl methyl ether) (PVME) from broadband dielectric spectroscopy are employed. ${ }^{28,29}$ We have also assumed $\beta_{0}=0.5$, that - as will be shown later in this paper-is the high temperature limit of $\beta_{\mathrm{KWw}}$ for this polymer, and the standard deviation of Vogel temperatures is fixed at: $\delta T_{0}=2.3 \mathrm{~K}$. The KWW description is a good approximation of Eq. (5) with a Gaussian distribution of Vogel temperatures, as testified by the excellent fits of the relaxation functions with the KWW 


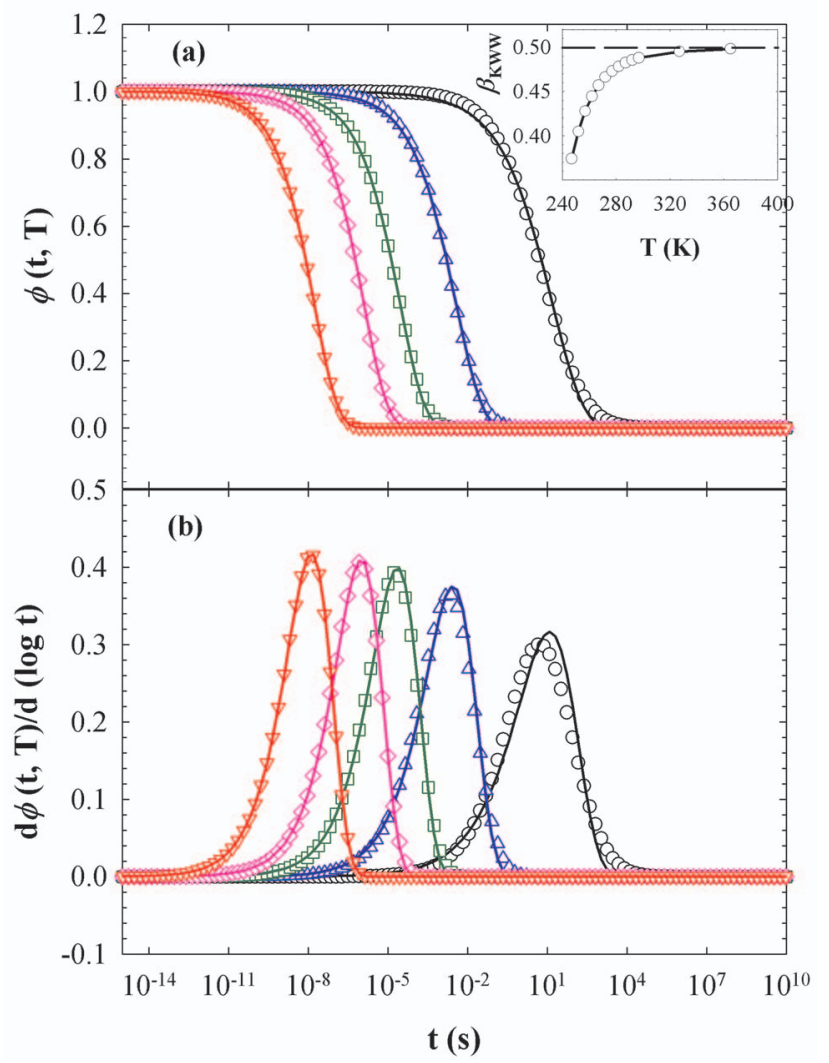

FIG. 1. (Color) Time evolution of the relaxation function (a) and of its derivative with the logarithm of time (b) for PVME evaluated through Eq. (5) (straight lines) at the following temperatures: $247 \mathrm{~K}$ (black), $262 \mathrm{~K}$ (blue), $277 \mathrm{~K}$ (green), $292 \mathrm{~K}$ (pink), and $327 \mathrm{~K}$ (red). The following VFT parameters were used: $T_{0(\mathrm{av})}=205 \mathrm{~K}, D=6.4$, and $\tau_{0}=3.8 \times 10^{-13} \mathrm{~s}$ taken from Refs. 28 and 29 where PVME dielectric relaxation data are provided; the intrinsic stretching exponent and the standard deviation of the distribution of Vogel temperatures: were, respectively, $\beta_{0}=0.5$ and $\delta T_{0}=2.3 \mathrm{~K}$. The points are the fitting of the relaxation functions through the KWW equation. The inset displays the temperature evolution of the stretching exponent (line and circle) and the limiting value of the stretching exponent (dashed line).

equation. The inset of Fig. 1 displays the resulting temperature dependence of the stretching exponent $\beta_{\mathrm{KWw}}$. This exponent decreases with decreasing temperature, qualitatively mimicking the temperature dependence of $\beta_{\mathrm{KWW}}$ normally observed experimentally, despite the assumed temperature independence of $\delta T_{0}$, quantifying the degree of dynamical heterogeneity. Furthermore, it is noteworthy that for $T / T_{g}$ $>1.2$ the stretching exponent $\beta_{\mathrm{KWw}}$ assumes an almost constant value and, within the experimental uncertainty, equal to $\beta_{0}$.

In the way it has been developed, the approach followed provides a link between the temperature dependence of the relaxation time, expressed through the VFT equation, and the $\mathrm{KWW}$ exponent. It is therefore possible to check what is the effect of changing the characteristics of the relaxation time on the temperature dependence of $\beta_{\mathrm{KWw}}$. For what concerns the effect of changing the standard deviation $\delta T_{0}$ on the KWW exponent, in Fig. 2 we have plotted $\beta_{\mathrm{KWw}}$ versus the temperature at various $\delta T_{0}$. From the inspection of the figure, it is clear that an increase in $\delta T_{0}$ produces a stronger temperature dependence of $\beta_{\mathrm{KWw}}$. This implies that the stretching exponent at $T_{g}$ decreases with increasing $\delta T_{0}$, as shown

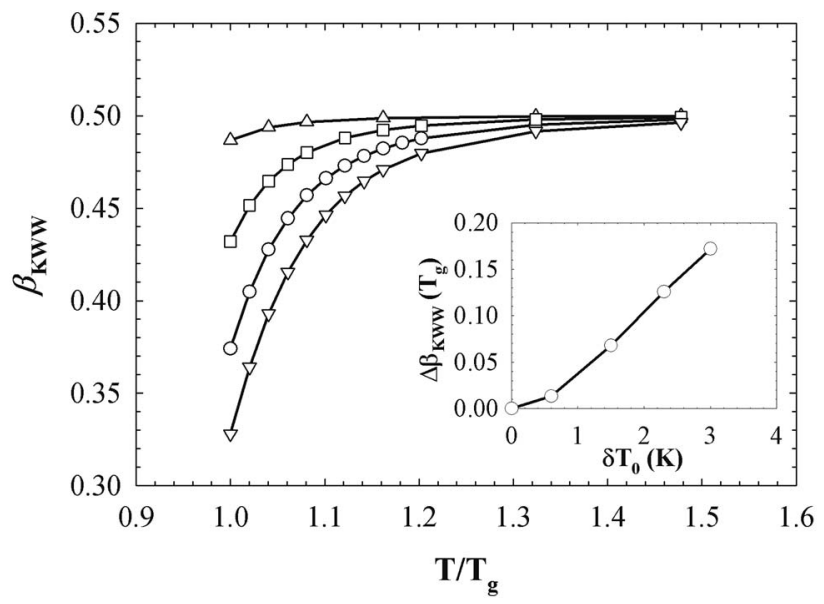

FIG. 2. Temperature dependence of the stretching exponent as a function of the standard deviation of the distribution of Vogel temperatures: (up triangles) $\delta T_{0}=0.6 \mathrm{~K}$; (squares) $\delta T_{0}=1.5 \mathrm{~K}$; (circles) $\delta T_{0}=2.3 \mathrm{~K}$; and (down triangles) $\delta T_{0}=3 \mathrm{~K}$. The VFT parameters and $\beta_{0}$ are like in Fig. 1 and the $T_{g}$ is defined as the temperature at which the relaxation time is: $\tau=100 \mathrm{~s}$. The inset displays the dependence of $\Delta \beta_{\mathrm{KWW}}=\beta_{0}-\beta_{\mathrm{KWW}}$, i.e., the deviation of the stretching exponent from the high temperature limit, at $T_{g}$ on $\delta T_{0}$.

in the inset of Fig. 2 where $\Delta \beta_{\mathrm{KWw}}=\beta_{0}-\beta_{\mathrm{KWw}}$, i.e., the deviation of the stretching exponent from the high temperature limit is presented. Furthermore, a relatively small $\delta T_{0}$ is not able to generate any significant decrease of $\beta_{\mathrm{KWW}}$ with decreasing temperature, as shown in Fig. 2 when fixing the standard deviation of Vogel temperature at: $\delta T_{0}=0.6 \mathrm{~K}$. This implies that, within our approach, a heterogeneity larger than $\delta T_{0} \approx 1 \mathrm{~K}$ is necessary to achieve a temperature dependent stretching of the relaxation function. Consequently, glassforming liquids whose dynamics verify the time-temperature superposition $\left(\beta_{\mathrm{KWw}}=\mathrm{const}\right)$ are expected to display a narrow distribution of Vogel temperatures. Remarkably the observation that the stretching exponent $\beta_{\mathrm{KWW}}$ assumes an almost constant value above $T / T_{g}>1.2$ almost equal to $\beta_{0}$ holds even for relatively large values of $\delta T_{0}$. This implies that in this temperature region the effect of heterogeneities on $\beta_{\mathrm{KWW}}$ can be considered negligible and the stretching of the relaxation function should be rather attributed to the intrinsic nature of the relaxation. ${ }^{16}$

The effect of the fragility on the stretching exponent is presented in Fig. 3. The former quantity, measuring the rapidity of the variation of the relaxation time with temperature at $T_{g}$, has been expressed in terms of the steepness index $(m)$ defined by the following equation: ${ }^{4,30}$

$$
m=\left.\frac{d \log _{10}\langle\tau\rangle}{d\left(T_{g} / T\right)}\right|_{P, T=T_{g}} .
$$

If the relaxation time is expressed according to the VFT equation [Eq. (2)], Eq. (6) can be rewritten as

$$
m=\frac{D}{\ln 10} \frac{T_{0(\text { av })} T_{g(\text { av })}}{\left(T_{g(\text { av })}-T_{0(\text { av })}\right)^{2}} .
$$

In Fig. 3, we report the temperature dependence of the stretching exponent $\beta_{\mathrm{KWW}}$ for various values of the steepness index. The fragility of the glass former has clearly a marked effect on the degree of stretching, being the latter enhanced, 


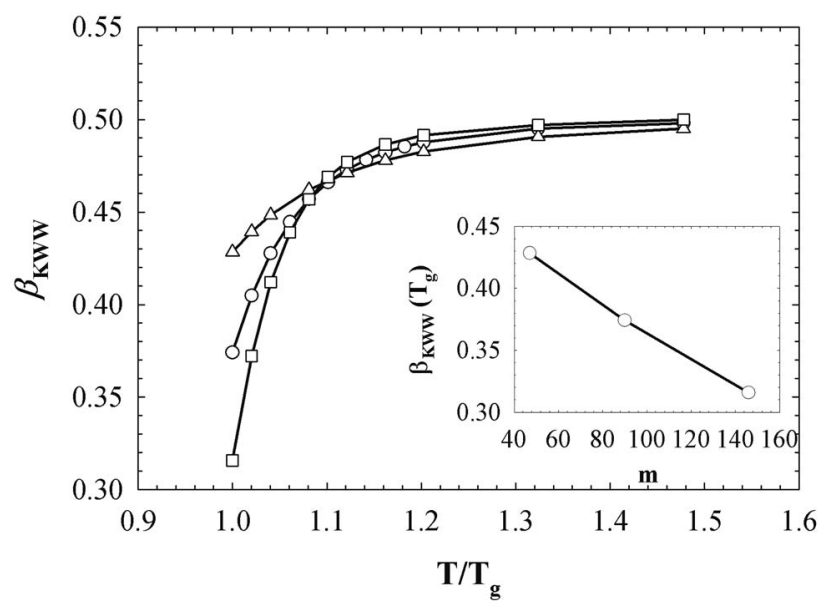

FIG. 3. Temperature dependence of the stretching exponent as a function of the steepness index: (up triangles) $m=47$; (circles) $m=90$; and (squares) $m=146$. The VFT parameters are chosen in a way that the system with $m=90$ corresponds to PVME. Those corresponding to the other steepness indices are chosen to have the same $T_{g}$ as the system with $m=90 . \delta T_{0}$ and $\beta_{0}$ are like in Fig. 1 and the $T_{g}$ is defined as the temperature at which the relaxation time is $\tau=100 \mathrm{~s}$. The inset displays the dependence of the stretching exponent at $T_{g}$ on the steepness index.

overall close to $T_{g}$ (see inset of Fig. 3), the larger the steepness index. This means that our approach is able to account for qualitative rough relation between $\beta_{\mathrm{KWW}}$ and the fragility often reported for fragile glass formers. ${ }^{4}$ In particular, when the fragility is allowed to vary while keeping all other parameters of the glass former fixed $\left(T_{g}, \beta_{0}, \log \tau_{0}\right), \beta_{\mathrm{KWW}}$ at $T_{g}$ scales with the fragility, being smaller the higher the fragility. It is, however, worth remarking that, within our approach, the fragility is not the only factor affecting $\beta_{\mathrm{KWW}}$, since - as already mentioned-also $\delta T_{0}$ and $\beta_{0}$ play a major role in affecting both the absolute value and temperature dependence of the stretching parameter. This may explain why the empirical correlation between $m$ and $\beta_{\mathrm{KWw}}$ (Ref. 4) is not universally verified for glass formers with very different chemical structures, as will be shown in details later in the paper (cf. Fig. 6). Notably, other approaches highlight the incapability of the stretching exponent to fully govern the fragility. ${ }^{31}$

To summarize this section, we remark that in the way we have approached the problem, the temperature dependence of the stretching exponent $\beta_{\mathrm{KWw}}$ arises in a natural way from the temperature dependence of the relaxation time. An essential hypothesis made is the presence of a distribution of relaxation times that has been expressed as a distribution of Vogel temperatures. This hypothesis—based on the assumption of a heterogeneous nature of the liquid state-relies on experimental evidences actually showing the presence of spatial heterogeneities with a lifetime that is of the order or larger than the structural relaxation time. ${ }^{11,13,15,20}$ However, we highlight that in our approach such heterogeneities are not the unique source of stretching, since we also assume intrinsic nonexponential dynamics. It is worth remarking that the hypothesis of a distribution of relaxation times is not new and is normally assumed $a$-priori in several models describing the nonexponentiality of the relaxation in glass-forming liquids. ${ }^{32-37}$ However, to the best of our knowledge, a straightforward connection between the temperature dependence of the relaxation time-in this case by means of the VFT equation-and the stretching exponent of the KWW equation through a distribution of relaxation times has not been presented yet. Such a connection and its test on experimental data represent the main scope of this work. In addition, the purpose of this paper is to raise questions about the temperature evolution of dynamical heterogeneities and its relation with some peculiar phenomena appearing below $1.2 T_{g}$ such as the decoupling between rotational and translational diffusion and that between the diffusion and the viscosity, ${ }^{38,39}$ that respectively violate the Debye and the Stokes-Einstein equations normally verified for simple liquids.

\section{EXPERIMENTAL DATA}

In this section, we report experimental data available in literature that have been tested within the proposed approach. We divide glass-forming liquids in two classes: polymeric and nonpolymeric glass formers. To apply the approach, the knowledge of the VFT parameters and that of the high temperature limit of the stretching exponent is required. The KWW relaxation time was employed to fit the VFT equation. These data are summarized in Table I where the reference from which data were taken is indicated. All data are from broadband dielectric spectroscopy except for the high temperature values of OTP that are from Fabry-Pérot interferometry. ${ }^{24}$ However, these data nicely match with those from dielectric spectroscopy obtained for OTP at lower temperature. ${ }^{24}$ In Table I, we also report the value of $T_{g}$ defined as the temperature where the relaxation time is $100 \mathrm{~s}$. In several cases dynamics data are obtained from broadband dielectric spectroscopy. This technique provides the complex dielectric permittivity $\varepsilon^{*}$ obtained in the frequency domain and normally fitted through the Havriliak-Negami ${ }^{40}(\mathrm{HN})$ phenomenological equation:

$$
\varepsilon^{*}(\omega)=\varepsilon_{\infty}+\frac{\Delta \varepsilon}{\left[1+\left(i \omega \tau_{\mathrm{HN}}\right)^{\alpha}{ }_{\mathrm{HN}}\right]^{\gamma_{\mathrm{HN}}}},
$$

where $\tau_{\mathrm{HN}}$ is the $\mathrm{HN}$ relaxation time, and $\alpha_{\mathrm{HN}}$ and $\gamma_{\mathrm{HN}}$ are the shape parameters of the HN function describing, respectively, the symmetric and asymmetric broadenings of the complex dielectric permittivity. These parameters are related to the stretching of the dipolar correlation function and, therefore, to the stretching exponent $\beta_{\mathrm{KWw}}$. This can be evaluated from the shape parameters of the $\mathrm{HN}$ function through the approach proposed by Alvarez et al. ${ }^{41}$ This approach also allows extracting the KWW relaxation time from the $\mathrm{HN}$ one.

For some glass formers, two sets of VFT parameters are reported (see Table I). This is due to the presence of a dynamic crossover, which can be effectively detected by means of the derivative analysis proposed several years ago by Stickel et al. ${ }^{42}$ As will be shown in Sec. IV of this paper, the employment of two VFT equations results in a crossover in the temperature dependence of $\beta_{\mathrm{KWw}}$.

Among the glass-forming polymers employed to perform the analysis, we have included also polyisoprene (PI). 
TABLE I. Relevant parameters and references for all investigated glass formers.

\begin{tabular}{|c|c|c|c|c|c|c|c|c|}
\hline Glass former & $\begin{array}{c}T_{g} \\
(\mathrm{~K})\end{array}$ & $\begin{array}{c}T_{0} \\
(\mathrm{~K})\end{array}$ & $D$ & $\begin{array}{c}\log _{10} \tau_{0} \\
(\mathrm{ps})\end{array}$ & $\beta_{0}$ & $m$ & Reference & $\begin{array}{l}\delta T_{0} \\
(\mathrm{~K})\end{array}$ \\
\hline Polyisoprene (PI) & 209 & 171 & 6.7 & -12.2 & 0.45 & 71.5 & 43 , this work & $<1$ \\
\hline Polybutadiene $^{\mathrm{a}}(\mathrm{PB})$ & 233.5 & 205 & 3.5 & -10.2 & 0.55 & 92 & 28 & 2.5 \\
\hline Poly(vinyl ethylene) (PVE) & 272 & 245 & 3 & -10.9 & 0.5 & 118 & 28 & 2.5 \\
\hline Phenoxy $^{\mathrm{b}}(\mathrm{PH})$ & 371 & 338 & 3 & -12.6 & 0.5 & 151 & 28 & 2 \\
\hline Poly(vinyl isobutylether) (PViBE) & 240.5 & 185 & 9.1 & -12.3 & 0.5 & 57 & 46 & 4 \\
\hline Poly(vinyl methylether) (PVME) & 244 & 205 & 6.4 & -12.4 & 0.5 & 90 & 28 & 2.3 \\
\hline Polysulfone (PSF), $T<475 \mathrm{~K}$ & 461.5 & 377 & 10.6 & -20.8 & 0.75 & 112 & 28 & 2.7 \\
\hline Polysulfone (PSF), $T>475 \mathrm{~K}$ & & 437.5 & 1.4 & -10.35 & & & & 2.5 \\
\hline Glycerol & 188 & 131 & 16.8 & -14.7 & 0.75 & 55 & 45,46 & 1.2 \\
\hline Propylene carbonate (PC), $T<200 \mathrm{~K}$ & 157 & 132 & 6.8 & -13.9 & 0.93 & 100 & 42,47 & 1.2 \\
\hline Propylene Carbonate (PC), $T>200 \mathrm{~K}$ & & 153 & 2.4 & -11.5 & & & & 1.6 \\
\hline$o$-terphenyl (OTP), $T<290 \mathrm{~K}$ & 247 & 172 & 22.1 & -21.1 & 1 & 74 & 48,24 & 1.8 \\
\hline$o$-terphenyl (OTP), $T>290 \mathrm{~K}$ & & 235 & 2.2 & -11.6 & & & & 3.4 \\
\hline Salol, $T<265 \mathrm{~K}$ & 222 & 166.5 & 15.2 & -18.8 & 1 & 79 & 42,45 & 2.4 \\
\hline Salol, $T>265 \mathrm{~K}$ & & 224 & 1.5 & -11.2 & & & & 4 \\
\hline $\mathrm{BCDE}^{\mathrm{c}}, T<270 \mathrm{~K}$ & 245.5 & 128.5 & 64 & -29.8 & 0.62 & 65 & 48,45 & 1 \\
\hline $\mathrm{BCDE}^{\mathrm{c}}, T>270 \mathrm{~K}$ & & 184.5 & 14.4 & -18 & & & & 1.5 \\
\hline $\mathrm{BKDE}^{\mathrm{d}}{ }^{\mathrm{d}} T<323 \mathrm{~K}$ & 262 & 212 & 9 & -14.5 & 0.66 & 87 & 45 & 2.5 \\
\hline $\mathrm{BKDE}^{\mathrm{d}}, T>323 \mathrm{~K}$ & & 269 & 2 & -11.4 & & & & 2.7 \\
\hline $\mathrm{PDE}^{\mathrm{e}} T>321 \mathrm{~K}$ & 293.5 & 162 & 62 & -31 & 0.96 & 74 & 49 & 0.9 \\
\hline $\mathrm{PDE}^{\mathrm{e}} T>321 \mathrm{~K}$ & & 280 & 2.5 & -11 & & & & 1.5 \\
\hline Benzophenone, $T<235 \mathrm{~K}$ & 208 & 100 & 96 & -36.5 & 0.8 & 74 & 50 & 0.7 \\
\hline Benzophenone, $T>235 \mathrm{~K}$ & & 198.5 & 2.8 & -12 & & & & 1.8 \\
\hline
\end{tabular}

${ }^{\mathrm{a}} 68 \% 1,2$ units and $32 \% 1,4$ units.

${ }^{\mathrm{b}} \mathrm{Poly}(2$-hydroxy propyl ether of bisphenol-A).

${ }^{\mathrm{c}}$ Bisphenol-C-dimethylether.

${ }^{\mathrm{d}}$ Dimethylbisphenol-C-dimethylether.

${ }^{\mathrm{e}}$ Phenylphthalein-dimethylether.

For this polymer, we have performed high frequency dielectric measurements on the same PI recently employed for low frequency dielectric experiments. ${ }^{43}$ Our results generally agree with those recently obtained by Doxastakis et al. ${ }^{44}$ Polyisoprene was purchased from Polymer Source Inc. The molecular weight was $M_{n}=3 \times 10^{4} \mathrm{~g} / \mathrm{mol}\left(M_{w} / M_{n}=1.04\right)$ and the sample was used as received. Dielectric measurements were carried out using an Agilent impedance analyzer HP4291B (frequency range of $10^{6}-10^{9} \mathrm{~Hz}$ ). A Novocontrol Quatro cryosystem for temperature control with a precision of $0.1 \mathrm{~K}$ was employed in all measurements.

\section{DATA ANALYSIS AND DISCUSSION}

Figures 4 and 5 display all experimental stretching exponents, respectively, for polymeric and nonpolymeric glass formers as a function of the temperature normalized at the $T_{g}$. Data are taken from the references indicated in Table I. The lines in the figures are the fits through our approach to experimental data. The values of $\delta T_{0}$ providing such fit of the experimental data are reported in Table I. Considering that experimental stretching exponents are always subjected to a certain degree of uncertainty (normally \pm 0.05 ), the fits can be considered in all cases more than satisfactory.

It is noteworthy that, for those glass formers displaying a dynamic crossover detectable through the Stickel analysis, ${ }^{42}$ two values of $\delta T_{0}$, one for each dynamic range of the glass former, are provided in Table I. The two values are not independent from each other as the relaxation times arising from the two VFT equations, describing the dynamics of the glass former in the whole temperature range, must be equal at the crossing temperature $(\bar{T})$. Thus, these two values of $\delta T_{0}$ are related to each other by the following relation:

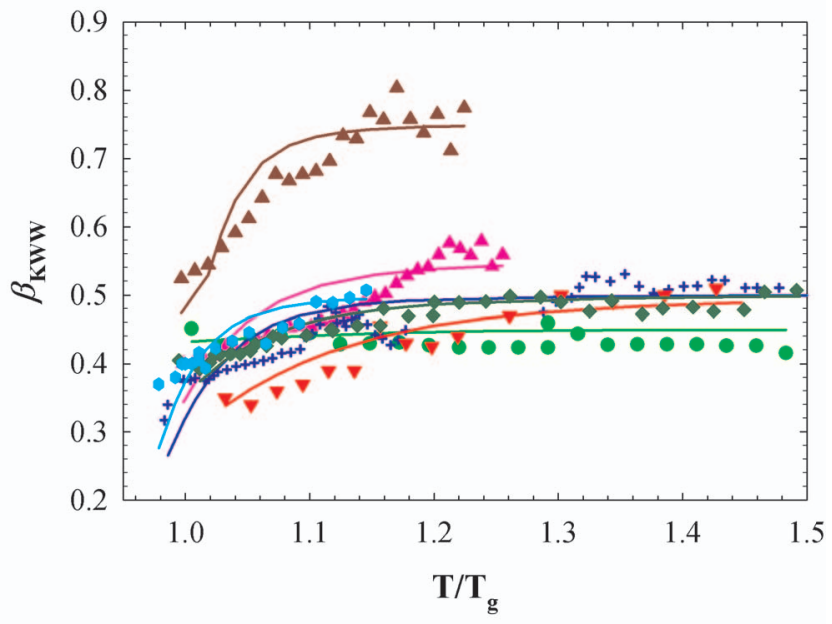

FIG. 4. (Color) Temperature dependence of the stretching exponent for the following polymeric glass formers: PI (light green circles); PB (pink up triangles); PVE (blue crosses); PViBE (red down triangles); PVME (dark green diamonds); PSF (brown up triangles); and PH (light blue circles). Continuous lines are the fits of our approach to experimental data with the parameters indicated in Table I. 


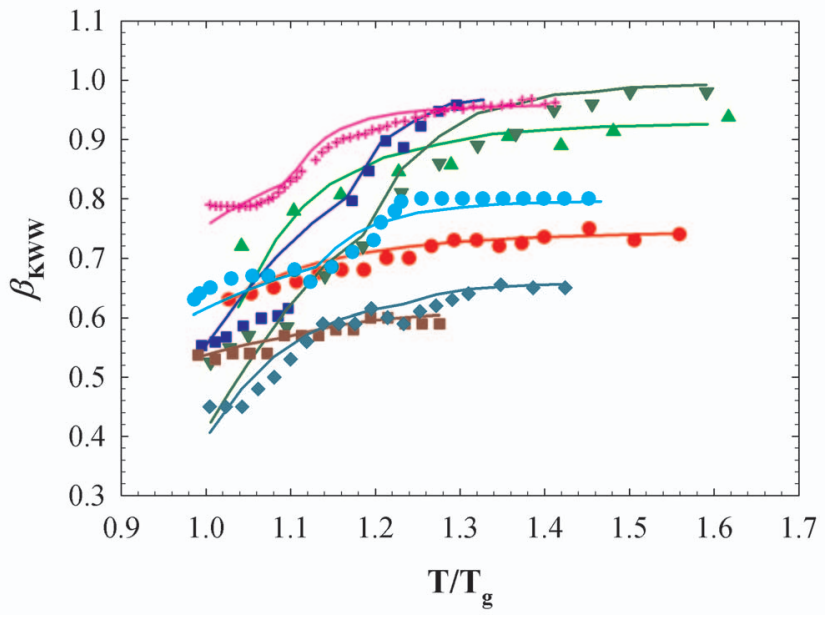

FIG. 5. (Color) Temperature dependence of the stretching exponent for the following nonpolymeric glass formers: glycerol (red circles); PC (light green up triangles); OTP (blue squares); salol (green down triangles); BCDE (brown squares); BKDE (dark cyan diamonds); PDE (pink crosses); and Benzophenone (light blue circles). Continuous lines are the fits of our approach to experimental data with the parameters indicated in Table I.

$$
\begin{aligned}
\tau_{0(1)} & \exp \left[\frac{D_{1}\left(T_{0(\mathrm{av})(1)} \pm \delta T_{0(1)}\right)}{\bar{T}-\left(T_{0(\mathrm{av})(1)} \pm \delta T_{0(1)}\right)}\right] \\
= & \tau_{0(2)} \exp \left[\frac{D_{2}\left(T_{0(\mathrm{av})(2)} \pm \delta T_{0(2)}\right)}{\bar{T}-\left(T_{0(\mathrm{av})(2)} \pm \delta T_{0(2)}\right)}\right]
\end{aligned}
$$

where the suffixes 1 and 2 are used to label the low and the high temperature dynamic ranges.

In the way we have related the stretching exponent to the relaxation time, it is interesting to notice that the dynamic crossover observed for all low molecular weight glass formers but glycerol, and for PSF reflects in an analogous crossover in the calculated stretching exponent. Interestingly, such a discontinuity is in most of the cases also observed in the experimental stretching exponent (see Figs. 4 and 5). This result implies that the temperature dependence of the stretching exponent and that of the relaxation time are related to each other. ${ }^{4,28}$ This experimental aspect is nicely captured by our approach that is actually based on the relation between the stretching exponent and the relaxation time.

Regarding the values of the standard deviations of the distribution of Vogel temperatures $\delta T_{0}$, these are comprised between 1 and $4 \mathrm{~K}$ for all glass formers. This implies thatalthough the correspondence between the dispersion of $T_{g}$ and that of $T_{0}$ is not exact-the values of $\delta T_{0} \approx \delta T_{g}$ obtained through our approach are highly realistic from a physical point of view. The only notable exception for the magnitude of $\delta T_{0}$ is PI whose dynamical response experimentally verifies the time-temperature superposition, i.e., the stretching exponent is essentially temperature independent. As this glass-forming polymer presents a medium fragility ( $m$ $=71.5$ ), the only way to explain this result according to our approach is the assumption of a low intrinsic heterogeneity associated to this polymer $\delta T_{0}<1 \mathrm{~K}$.

In order to quantify the structural implications of the obtained values of the standard deviations of the distribution

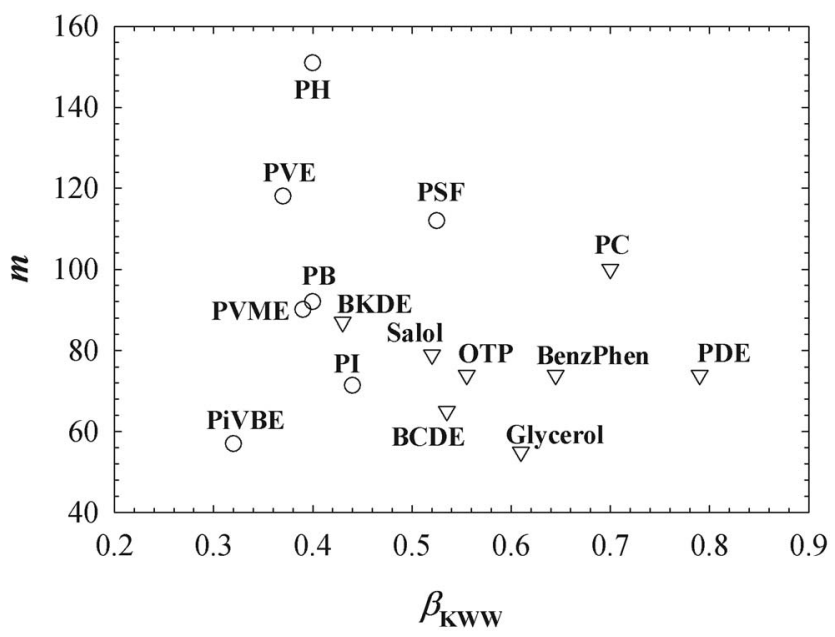

FIG. 6. Steepness index vs stretching exponent at $T_{g}$ for polymeric (empty circles) and nonpolymeric (empty triangles) glass formers.

of Vogel temperatures, such values can be converted into the associated local density distribution. This can be done inspecting the effect of pressure, and therefore of density change, on the variation of the Vogel temperature. Taking PVME as an example, a shift of the Vogel temperature of 2.3 $\mathrm{K}$, equal to the $\delta T_{0}$ determined for this polymer, can be achieved applying a pressure of $P=13.5 \mathrm{MPa},{ }^{51}$ which, in turns, corresponds to an increase in the density of $\Delta \rho$ $=0.56 \% .^{52}$ This means that the obtained $\delta T_{0}$ would be generally associated to density changes smaller than $1 \%$. Such small changes would be consistent with the absence of any clear signature of structural heterogeneities observed by small angle $\mathrm{x}$-ray scattering experiments in proximity of the $T_{g}{ }^{53}$

In the context of the connection between the fragility and the stretching exponent at $T_{g}$, our approach provides a rationalization of this connection as highlighted earlier in this paper. However, it is worth noticing that the value of the stretching exponent at $T_{g}$ is not solely related to the fragility of the glass former, being the high temperature value of the stretching exponent and the heterogeneity of the system essential ingredients affecting the breadth of the relaxation at $T_{g}$. This might be the reason for the poor inverse relation between the steepness index $m$ and $\beta_{\mathrm{KWW}}$, even failing for some glass formers. ${ }^{4}$ When these two parameters are displayed in the same plot for the glass formers under investigation, the aforementioned inverse correlation is hardly found, as shown in Fig. 6. This generally indicates that other variables should be responsible for the value of $\beta_{\mathrm{KWW}}$ at $T_{g}$. As a notable example, poly(vinyl isobutylether) (PViBE), possessing a rather low fragility $(m=57)$ can achieve a stretching exponent at $T_{g}$ as low as $\beta_{\mathrm{KWW}}=0.32$ only if a large intrinsic heterogeneity is assumed. The $\delta T_{0}$ of PViBE is actually found to be the largest among the investigated glass formers $\left(\delta T_{0}=4 \mathrm{~K}\right)$.

An important implication of the ability of temperature independent heterogeneities in generating a decreasing $\beta_{\mathrm{KWW}}$ with decreasing temperature is that those typical signatures marking the approach to the glass transition, like the decoupling between rotational and translational diffusion and 
that between the diffusion and the viscosity, ${ }^{38,39}$ might not bear any relation with the temperature behavior of the stretching exponent. As a prominent example we mention that of OTP, which does not present a significant change of the stretching exponent in proximity of the $T_{g}{ }^{7}$ despite the marked decoupling between translational diffusion and viscosity. ${ }^{54}$

\section{CONCLUSIONS}

We have presented an approach able to quantitatively reproduce the decrease in the stretching exponent of the KWW equation with decreasing temperature. According to our approach an intrinsic nonexponential behavior in combination with temperature independent heterogeneities is able to quantitatively describe the temperature dependence of the stretching exponent for a large variety of glass formers. The approach followed also involves the employment of the VFT equation and, therefore, provides a link between the stretching exponent and the structural relaxation time. The assumption of temperature independent heterogeneities implies that the decrease in the stretching exponent $\beta_{\mathrm{KWw}}$ with decreasing temperature might bear no relation with the often assumed increase of the heterogeneity of the glass former. Therefore, one of the main conclusions of this work is that any interpretation of the temperature dependence of the stretching exponent $\beta_{\mathrm{KWw}}$ in terms of temperature evolution of heterogeneities-expressed as a distribution of Vogel temperatures-in the liquid state should be, according to our analysis, reconsidered.

Furthermore, within our approach, the rapidity of the $\beta_{\mathrm{KWw}}$ decrease with decreasing temperature is a function of the degree of heterogeneity, i.e., the value of $\delta T_{0}$, the fragility of the system, namely the value of the steepness index $m$, and $\beta_{0}$. In particular, the temperature dependence of $\beta_{\mathrm{KWW}}$ turns out to be more marked when $\delta T_{0}$ and $m$ are larger. As, according to the latter correlation, relatively low values of the stretching exponent $\beta_{\mathrm{KWW}}$ at $T_{g}$ are expected for highly fragile glass formers, our approach is able to rationalize the empirical correlation of Bohmer et al. ${ }^{4}$ between $\beta_{\mathrm{KWW}}$ and $m$. Nonetheless, we have highlighted the inability of the steepness index $m$ to be entirely decisive in affecting the degree of stretching at $T_{g}$, being the intrinsic stretching of the glass former and the magnitude of $\delta T_{0}$ the other significant ingredients.

\section{ACKNOWLEDGMENTS}

The authors acknowledge the University of the Basque Country and Basque Country Government (Ref. No. IT-43607, Depto. Educación, Universidades e Investigación) and the Spanish Minister of Education (Grant No. MAT 200763681) for their support. The support of the European Community within the SOFTCOMP program was also acknowledged.

\footnotetext{
${ }^{1}$ R. Kohlrausch, Ann. Phys. Chem. 167, 179 (1854).

${ }^{2}$ G. Williams and D. C. Watts, Trans. Faraday Soc. 66, 80 (1970).

${ }^{3}$ Materials and Disordered Solids, An Introduction to Their Statistical Mechanics, edited by K. Binder and W. Kob (World Scientific, Singapore, 2005).
}

${ }^{4}$ R. Bohmer, K. L. Ngai, C. A. Angell, and D. J. Plazek, J. Chem. Phys. 99, 4201 (1993).

${ }^{5}$ Relaxation Phenomena in Polymers, edited by S. Matsuoka (Hanser, Munich, 1992); Glass: Structure by Spectroscopy, edited by J. Wong and C. A. Angell (Dekker, New York, 1976).

${ }^{6}$ Viscoelastic Properties of Polymers, edited by J. D. Ferry (Wiley, New York, 1980).

${ }^{7}$ R. Richert, J. Chem. Phys. 123, 154502 (2005).

${ }^{8}$ C. Maggi, B. Jakobsen, T. Christensen, N. B. Olsen, and J. C. Dyre, J. Phys. Chem. B 112, 16320 (2008).

${ }^{9}$ Anelastic and Dielectric Effects in Polymeric Solids, edited by N. G. McCrum, B. E. Read, and G. Williams (Dover, New York, 1991).

${ }^{10}$ H. Sillescu, J. Non-Cryst. Solids 243, 81 (1999).

${ }^{11}$ M. D. Ediger, Annu. Rev. Phys. Chem. 51, 99 (2000)

${ }^{12}$ S. C. Glotzer, J. Non-Cryst. Solids 274, 342 (2000).

${ }^{13}$ R. Richert, J. Phys.: Condens. Matter 14, R703 (2002).

${ }^{14}$ Fifth International Discussion Meeting on Relaxations in Complex Systems [ J. Non-Cryst. Solids 352, 4371 (2006)]; Fourth Workshop on Non-equilibrium Phenomena in Supercooled Fluids, Glasses and Amorphous Materials [ J. Phys.: Condens. Matter 19, 200301 (2007)].

${ }^{15}$ K. Schmidt-Rohr and H. W. Spiess, Phys. Rev. Lett. 66, 3020 (1991).

${ }^{16}$ A. Arbe, J. Colmenero, M. Monkenbusch, and D. Richter, Phys. Rev. Lett. 81, 590 (1998).

${ }^{17}$ H. Vogel, Phys. Z. 22, 645 (1921); G. S. Fulcher, J. Am. Chem. Soc. 8, 339 (1925).

${ }^{18}$ R. G. Palmer, D. L. Stein, E. Abrahams, and P. W. Anderson, Phys. Rev. Lett. 53, 958 (1984).

${ }^{19}$ W. Götze and L. Sjögren, Rep. Prog. Phys. 55, 241 (1992).

${ }^{20}$ R. Bohmer, G. Diezemann, G. Hinze, and E. Rossler, Prog. Nucl. Magn. Reson. Spectrosc. 39, 191 (2001).

${ }^{21}$ The stretching exponent obtained in the microwaves frequency range by dielectric spectroscopy can be generally perceived as its high temperature limit. Such a conclusion arises from the general agreement between the stretching exponent obtained in the microwaves region and that obtained at much shorter time higher temperatures by molecular dynamics simulation. See, for instance, S. Mossa, R. Di Leonardo, G. Ruocco, and M. Sampoli, Phys. Rev. E 62, 612 (2000); A. Adachi, E. Saiz, and E. Riande, Phys. Chem. Chem. Phys. 4, 635 (2002).

${ }^{22}$ G. Williams, Chem. Soc. Rev. 7, 89 (1978).

${ }^{23}$ Electrical Properties of Polymers, edited by E. Riande and R. DiazCalleja (Dekker, New York, 2004); Broadband Dielectric Spectroscopy, edited by F. Kremer and A. Schönhals (Springer, Berlin, 2003).

${ }^{24}$ W. Steffen, A. Patkowski, H. Glaser, G. Meier, and E. W. Fischer, Phys. Rev. E 49, 2992 (1994).

${ }^{25}$ A. Tölle, J. Wuttke, H. Schober, O. G. Randl, and F. Fujara, Eur. Phys. J. B 5, 231 (1998).

${ }^{26}$ M. Tyagi, A. Alegría, and J. Colmenero, J. Chem. Phys. 122, 244909 (2005).

${ }^{27}$ The loss part of the dielectric permittivity is normally presented when discussing dielectric relaxation data. Employing the Hamon method, the log-time derivative of the relaxation function is directly related to the loss part of the dielectric permittivity. For details, see V. B. Hamon, Proc. Instr. Electr. Eng. 99, 151 (1952).

${ }^{28}$ A. Alegría, J. Colmenero, P. O. Mari, and I. A. Campbell, Phys. Rev. E 59, 6888 (1999).

${ }^{29}$ I. Cendoya, A. Alegría, J. M. Alberdi, J. Colmenero, H. Grimm, D. Richter, and B. Frick, Macromolecules 32, 4065 (1999).

${ }^{30}$ A. C. Angell, J. Non-Cryst. Solids 131-133, 13 (1991).

${ }^{31}$ P. K. Gupta and J. C. Mauro, Phys. Rev. E 78, 062501 (2008).

${ }^{32}$ R. V. Chamberlin, Phys. Rev. B 48, 15638 (1993).

${ }^{33}$ C. Monthus and J. P. Bouchuad, J. Phys. A 29, 3847 (1996).

${ }^{34}$ G. Diezemann, J. Chem. Phys. 107, 10112 (1997).

${ }^{35}$ J. S. Langer and S. Mukhopadhyay, Phys. Rev. E 77, 061505 (2008).

${ }^{36}$ X. Xia and P. G. Wolynes, Phys. Rev. Lett. 86, 5526 (2001).

${ }^{37}$ J. P. Garrahan and D. Chandler, Phys. Rev. Lett. 89, 035704 (2002).

${ }^{38}$ F. Fujara, B. Geil, H. Sillescu, and G. Z. Fleischer, Z. Phys. B: Condens. Matter 88, 195 (1992).

${ }^{39}$ M. T. Cicerone and M. D. Ediger, J. Chem. Phys. 104, 7210 (1996).

${ }^{40}$ S. Havriliak and S. Negami, Polymer 8, 161 (1967).

${ }^{41}$ F. Alvarez, A. Alegría, and J. Colmenero, Phys. Rev. B 44, 7306 (1991).

${ }^{42}$ F. Stickel, E. W. Fischer, and R. Richert, J. Chem. Phys. 102, 6251 (1995). 
${ }^{43}$ D. Cangialosi, A. Alegría, and J. Colmenero, Macromolecules 39, 7149 (2006); S. Cerveny, P. Zinck, M. Terrier, S. Arrese-Igor, A. Alegría, and J. Colmenero, ibid. 41, 8669 (2008).

${ }^{44}$ M. Doxastakis, D. N. Theodorou, G. Fytas, F. Kremer, R. Faller, F. Muller-Plathe, and N. Hadjichristidis, J. Chem. Phys. 119, 6883 (2003).

${ }^{45}$ A. Hofmann, Ph.D. thesis, Mainz, 1993.

${ }^{46}$ A. Schonhals, F. Kremer, A. Hofmann, and E. W. Fischer, Physica A 201, 263 (1993).

${ }^{47}$ U. Schneider, P. Lunkenheimer, R. Brand, and A. Loidl, Phys. Rev. E 59, 6924 (1999).

${ }^{48}$ C. Hansen, F. Stickel, T. Berger, R. Richert, and E. W. Fischer, J. Chem Phys. 107, 1086 (1997).
${ }^{49}$ R. Casalini, K. L. Ngai, and C. M. Roland, Phys. Rev. B 68, 014201 (2003).

${ }^{50}$ P. Lunkenheimer, L. C. Pardo, M. Köhler, and A. Loidl, Phys. Rev. E 77, 031506 (2008).

${ }^{51}$ G. A. Schwartz, A. Alegría, and J. Colmenero, Macromolecules 39, 3931 (2006).

${ }^{52}$ R. Casalini and C. M. Roland, J. Chem. Phys. 119, 4052 (2003).

${ }^{53}$ J. H. Wendorff, E. W. Fischer, and Z. Z. Kolloid, Polymer 251, 884 (1973).

${ }^{54}$ M. K. Mapes, S. F. Swallen, and M. D. Ediger, J. Phys. Chem. B 110, 507 (2006). 